\title{
Perfil sociodemográfico e de hábitos de vida da população idosa de um município da região norte do Rio Grande do Sul, Brasil
}

\author{
Sociodemographic profile and lifestyle of the elderly population in a city in northern Rio Grande do \\ Sul state, Brazil
}

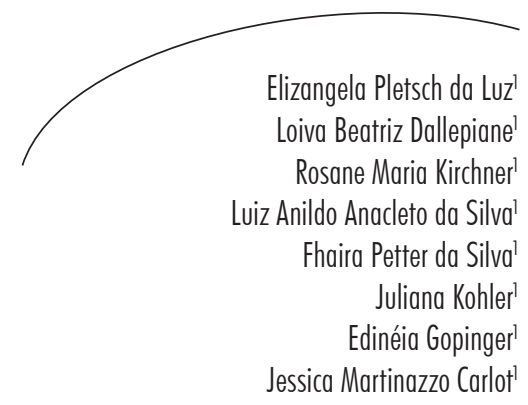

\section{Resumo}

Objetivo: Este estudo teve como objetivo caracterizar o perfil sociodemográfico e de hábitos de vida da população idosa de um município da região norte do Rio Grande do Sul, Brasil. Metodologia: Pesquisa quantitativa, descritiva e transversal. As variáveis analisadas foram: sociodemográficas (idade, sexo, estado civil, escolaridade e renda) e hábitos de vida (etilismo, tabagismo e atividade física). Resultados: Participaram da pesquisa 424 idosos, sendo 68,4\% do sexo feminino; a idade variou de 60 a 99 anos, com um número maior de idosos acima de 80 anos (15,6\%) do que dos 75 aos 80 anos $(14,6 \%)$; situação conjugal predominante foi "casado" (53,8\%); a maioria com menos de cinco anos de escolaridade (60,1\%). A renda familiar ficou entre um e dois salários mínimos, com dois dependentes. As principais despesas foram com alimentação, saúde/medicamentos, sendo que $75,7 \%$ fizeram uso de medicamentos diários e $71,3 \%$ consumiram de um a três medicamentos ao dia. A maioria não consumia bebidas alcoólicas (85,6\%), não tinha o hábito de fumar (85,6\%), 48,3\% dos idosos não praticavam atividade física regular. Conclusão: Com base nos resultados deste estudo, constatou-se que o perfil sociodemográfico predominante é de idosos de baixa renda e escolaridade; e com relação aos hábitos de vida, a maioria não ingere bebida alcoólica, não fuma, não pratica atividade física e suas principais despesas são com alimentação e saúde/medicamentos. Neste contexto, entende-se que a gestão pública de saúde tem um grande desafio com o envelhecimento, dentre eles, capacitar técnicos e profissionais, desenvolver políticas inclusivas, valorizando mais a pessoa do idoso, assim como seus cuidadores/família, oferecendo suporte adequado.

\section{Abstract}

Objective: This study aimed to characterize the sociodemographic profile of elderly in a city in northern Rio Grande do Sul state, Brazil. Methodology: Quantitative, descriptive and transversal research analyzing sociodemographic (age, sex, marital status, educational background and income) and lifestyle (alcoholism, smoking and physical exercise) variables. Results: Four hundred and twenty-four elderly people participated in

Centro de Educação Superior Norte-RS. Universidade Federal de Santa Maria. Palmeira das Missões, RS, Brasil.

Palavras-chave: Idoso. Nutrição. Saúde do idoso. Saúde Pública. 
the study, being 68.4\% women aged 60-69 years old. There were more individuals aged 80 than $75-80$ years (14.6\%); predomination of marital status "married" (53.8\%); most had studied for less than five years (60.1\%), had family income between one and two minimum wages, and with two dependent people. The main expenses were related to feeding, health/drugs, and $75.7 \%$ of them made use of daily drugs, while $71.3 \%$ took from one to three drugs daily. Most of them did not consume alcoholic (85.6\%), nor smoked $(85.6 \%)$, and $48.3 \%$ of the elderly did not practice regular physical exercises. Conclusions: Based on the results of this study, the prevailing sociodemographic profile in the elderly is low income and poorer education; concerning lifestyle, most did not drink alcoholic beverages, smoke, or practice physical activity, and their main expenditure was feeding and health/drugs. In this context, it is understood that public health management has great challenges with ageing, such as enabling technicians and professionals develop inclusive policies that value more the elderly, as well as their caregivers and relatives, by offering adequate support.

\section{INTRODUÇÃO}

No Brasil, a velocidade do processo de transição demográfica e epidemiológica, vivido pelo país nas últimas décadas, impõe desafios aos gestores e pesquisadores dos sistemas de saúde, com repercussões para a sociedade de forma geral, sobretudo num contexto de importante desigualdade social, pobreza e fragilidade das instituições.

A evolução do ritmo de envelhecimento da população é observada por meio do Índice de Envelhecimento (IE), comparativamente entre áreas geográficas e grupos sociais. Desde 1980, o Estado do Rio de Janeiro apresentou IE superior ao Rio Grande do Sul e somente no Censo de 2010 esta posição se inverteu, passando o Rio Grande do Sul a ocupar a primeira posição entre os estados brasileiros, e o Rio de Janeiro, a segunda. ${ }^{2} \mathrm{O}$ Rio Grande do Sul alcançou a condição de primeiro estado brasileiro em número proporcional de idosos (13,6\%), comparando com o Brasil, que é de $10,8 \%$, e o segundo lugar em expectativa de vida $(75,5$ anos $) .^{3}$

No contexto do envelhecimento humano, mobilizar-se significa criar ambientes comunitários seguros e bem projetados, a fim de apoiar comportamentos saudáveis que ajudam a prevenir doenças crônicas e lesões não intencionais, e permite que os idosos possam ser ativos e engajados na vida da comunidade o maior tempo possível. ${ }^{4}$
Key words: Elderly. Nutrition. Health of the Elderly. Public Health.
Para os idosos, poucas coisas são mais importantes do que a disponibilidade e acesso aos serviços de saúde de qualidade. Nesse sentido, a sociedade deve prover esforços de parcerias de setores públicos, privado e sem fins lucrativos, a fim de assegurar a saúde integral dos idosos. Esses esforços devem incluir educação para a saúde, exames, imunizações e aconselhamento sobre o uso de medicamentos, bem como programas de transição de cuidados para garantir que os pacientes após a saída de ambientes hospitalares, recebam o apoio necessário, a fim de evitar o risco de readmissão prematura. ${ }^{5}$

O fenômeno do envelhecimento populacional é uma questão de saúde pública que repercute nas diferentes esferas da estrutura social, econômica, política e cultural da sociedade, com demandas específicas e implicando mudanças nos diversos setores de atenção, público e privado. ${ }^{6}$ Dentre tantas preocupações para melhor atender à população acima dos 60 anos de idade, uma, em especial, é a formação adequada dos profissionais de saúde, para que estes estejam capacitados e preparados a compreender e trabalhar com as diversas situações e demandas próprias dessa faixa etária?

Alguns dispositivos legais auxiliam as ações sociais e de saúde, garantindo os direitos das pessoas idosas e exigindo do Estado a proteção dos mesmos. Dentre eles, podem ser citados a 
Política Nacional do Idoso, a Política Nacional de Saúde da Pessoa Idosa e o Estatuto do Idoso. ${ }^{8}$ Diante destas novas demandas, o envelhecimento é alvo privilegiado de investigações nas mais diferentes áreas de conhecimento: quanto mais informações e conhecimento se obtêm sobre determinada população, maiores as chances de intervenções bem-sucedidas, seja em conglomerados populacionais de pequeno ou grande porte. Porém, também é notório que a realização de políticas públicas efetivas vai muito além de conhecer a população e ter a melhor legislação disponível; exige a corresponsabilidade do Estado, dos profissionais das áreas de saúde, da assistência social, bem como do idoso e da sociedade em geral.

Nesta perspectiva, este estudo objetivou caracterizar o perfil sociodemográfico e de hábitos de vida de idosos de um município da região norte do Estado do Rio Grande do Sul (RS), Brasil.

\section{METODOLOGIA}

Trata-se de uma pesquisa quantitativa, descritiva e transversal, envolvendo indivíduos idosos residentes em um município da região norte do RS que possui 33.846 habitantes, sendo 4.037 idosos, correspondendo a 11,93\%. A amostra foi constituída de 424 idosos, selecionados ao acaso e seguindo a amostra calculada por Barbetta, ${ }^{9}$ considerando uma margem de erro de $5 \%$. Como critérios de inclusão, consideraram-se indivíduos com 60 anos de idade ou mais de ambos os sexos, sendo excluídos os idosos acamados. Os dados foram coletados no período de março de 2010 a dezembro de 2011.

As variáveis analisadas foram as sociodemográficas (idade, sexo, estado civil, escolaridade e renda) e de hábitos de vida (etilismo, tabagismo e atividade física). O etilismo, definido como o consumo regular de bebida alcoólica nos últimos 12 meses, foi considerado presente se igual ou superior a 30 gramas/álcool/dia, estimado pelo conteúdo alcoólico de cada tipo de bebida (duas garrafas de cerveja ou três copos de vinho ou duas doses de bebida destilada ou aguardente). ${ }^{10}$ O tabagismo foi definido como o hábito de fumar, independentemente do número e tipo de cigarros consumidos por dia, nos últimos cinco anos. ${ }^{11} \mathrm{~A}$ atividade física regular foi caracterizada por referências à prática de frequência semanal e duração diária de caminhadas, ginásticas e/ou hidroginástica, por período igual ou superior a seis meses no último ano. Foi considerada adequada quando realizada a frequência mínima de cinco vezes por semana e 30 minutos de duração para intensidades moderadas, três vezes por semana, e 20 minutos para as vigorosas, podendo estas serem complementares. O programa moderado pode ser acumulado em sessões de dez minutos, no mínimo. ${ }^{12}$

Os dados foram analisados utilizandose o software estatístico SPSS versão 18.0. A metodologia de análise foram a estatística descritiva e o teste de qui-quadrado.

A pesquisa seguiu as recomendações da Resolução no 196/96, do Conselho Nacional de Saúde, e foi aprovada pelo Comitê de Ética em Pesquisa da Universidade Federal de Santa Maria, processo no 23081.009908/2010-10. Após a concordância, todos os participantes assinaram o Termo de Consentimento Livre e Esclarecido.

\section{RESULTADOS}

Participaram da pesquisa 424 idosos, sendo $68,4 \%$ do sexo feminino, com idade variando

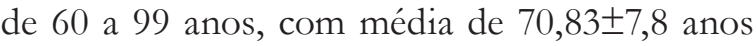
e predomínio na faixa etária de 65 a 70 anos (26,4\%). Quanto à situação conjugal, 53,8\% eram casados e $32,1 \%$ viúvos. Entre os idosos, 60,1\% tinham menos de cinco anos de escolaridade e renda familiar de um a dois salários mínimos $(68,4 \%)$. Desta renda familiar, oriunda da aposentadoria e/ou pensão, dependiam até duas pessoas num total de $80 \%$ dos casos, visto que $95,5 \%$ dos idosos estavam fora da população economicamente ativa (PEA). As principais despesas foram com alimentação $(52,6 \%)$, seguidas dos gastos com saúde/medicamentos $(38,2 \%)$, conforme a tabela 1 . 
Tabela 1. Características sociodemográficas dos idosos. Palmeira das Missões-RS, 2010-2011.

\begin{tabular}{|c|c|c|}
\hline Variáveis & $\mathrm{n}$ & $\%$ \\
\hline \multicolumn{3}{|l|}{ Sexo } \\
\hline Feminino & 290 & 68,4 \\
\hline Masculino & 134 & 31,6 \\
\hline \multicolumn{3}{|l|}{ Idade } \\
\hline $60 \mid-65$ & 101 & 23,8 \\
\hline $65 \mid-70$ & 112 & 26,4 \\
\hline $70 \mid-75$ & 83 & 19,6 \\
\hline $75 \mid-80$ & 62 & 14,6 \\
\hline 80 ou mais & 66 & 15,6 \\
\hline \multicolumn{3}{|l|}{ Estado civil } \\
\hline Solteiro & 29 & 6,8 \\
\hline Casado & 228 & 53,8 \\
\hline Viúvo & 136 & 32,1 \\
\hline Desquitado & 31 & 7,3 \\
\hline \multicolumn{3}{|l|}{ Escolaridade } \\
\hline Menos de cinco anos & 255 & 60,1 \\
\hline Cinco anos ou mais & 169 & 39,9 \\
\hline \multicolumn{3}{|l|}{ Renda } \\
\hline Menos de $1 \mathrm{SM}$ & 48 & 11,3 \\
\hline $1 \mathrm{a} 2 \mathrm{SM}$ & 290 & 68,4 \\
\hline 2 a $3 \mathrm{SM}$ & 51 & 12,0 \\
\hline 3 ou mais SM & 35 & 8,3 \\
\hline \multicolumn{3}{|l|}{ Dependentes da renda } \\
\hline $\mathrm{Um}$ & 127 & 30 \\
\hline Dois & 212 & 50 \\
\hline 3 a 5 & 77 & 18,1 \\
\hline 6 ou mais & 8 & 1,9 \\
\hline Não respondeu & 424 & 100 \\
\hline \multicolumn{3}{|l|}{ Fonte da renda } \\
\hline Aposentadoria e pensão & 381 & 89,9 \\
\hline Salário/aluguel/serviços/outros & 29 & 6,9 \\
\hline Não se aplica/não respondeu & 14 & 3,3 \\
\hline
\end{tabular}




\begin{tabular}{lcc}
\hline \multicolumn{1}{c}{ Variáveis } & $\mathrm{n}$ & $\%$ \\
\hline Principais despesas & 162 & 38,2 \\
Saúde/remédios & 31 & 7,3 \\
Habitação & 223 & 52,6 \\
Alimentação & 6 & 1,4 \\
Ajuda familiar & 1 & 0,2 \\
Vestuário & 1 & 0,2 \\
Não sabe/não respondeu & & \\
Ocupação & 405 & 95,5 \\
Indivíduo fora da PEA & 18 & 4,2 \\
Outros & 1 & 0,2 \\
Não respondeu & 424 & 100,0 \\
\hline Total & & \\
\hline
\end{tabular}

Na tabela 2, verifica-se a distribuição dos idosos em relação aos hábitos de vida, constatando-se que a maioria $(85,6 \%)$ não consumia bebidas alcoólicas. Entre os que consumiram, prevaleceu a categoria "raramente" (5,7\%), seguida de consumo "diário" e "semanal", na mesma proporção. O tipo de bebida mais consumida foi o vinho na quantidade de um copo. E ainda, 85,6\% dos idosos não tinham o hábito de fumar, mas entre os tabagistas $(14,4 \%)$, metade relatou fumar há mais de 35 anos.
Quanto à atividade física, 48,3\% dos idosos não tinham esta prática regular. No entanto, para os 45,3\% que praticavam, $13,7 \%$ relataram frequência de uma vez na semana, sendo a caminhada a atividade mais realizada e prevalecendo por mais de 45 minutos de duração, num período de mais de seis meses (35,8\%). Entre os idosos, 75,7\% fizeram uso de medicamentos diários, com o consumo de um a três medicamentos ao dia, conforme a tabela 2 .

Tabela 2. Hábitos de vida dos idosos. Palmeira das Missões-RS, 2010-2011.

\begin{tabular}{lcc}
\hline \multicolumn{1}{c}{ Variáveis } & $\mathrm{n}$ & $\%$ \\
\hline Consumo bebida alcoólica & 61 & 14,4 \\
Sim & 363 & 85,6 \\
Não & & \\
Frequência de consumo & 18 & 4,2 \\
Diariamente & 18 & 4,2 \\
Semanalmente & 1 & 0,2 \\
Mensalmente & 24 & 5,7 \\
Raramente & &
\end{tabular}




\begin{tabular}{|c|c|c|}
\hline Variáveis & $\mathrm{n}$ & $\%$ \\
\hline \multicolumn{3}{|l|}{ Quantidade ingerida } \\
\hline Vinho/copo & 33 & 7,8 \\
\hline Cerveja/copo & 22 & 5,2 \\
\hline Bebida destilada/dose & 6 & 1,4 \\
\hline \multicolumn{3}{|l|}{ Hábito de fumar } \\
\hline $\operatorname{Sim}$ & 61 & 14,4 \\
\hline Não & 363 & 85,6 \\
\hline \multicolumn{3}{|l|}{ Tempo de fumo } \\
\hline Menos de 5 anos & 6 & 1,4 \\
\hline 5 a 20 anos & 5 & 1,2 \\
\hline 20 a 35 anos & 7 & 1,7 \\
\hline Mais de 35 anos & 33 & 7,8 \\
\hline Não sabe & 10 & 2,4 \\
\hline \multicolumn{3}{|l|}{ Atividade física } \\
\hline $\operatorname{Sim}$ & 192 & 45,3 \\
\hline Não & 205 & 48,3 \\
\hline Não respondeu & 27 & 6,4 \\
\hline \multicolumn{3}{|l|}{ Frequência } \\
\hline Uma vez/semana & 58 & 13,7 \\
\hline Duas vezes/semana & 49 & 11,6 \\
\hline Três vezes/semana & 34 & 8,0 \\
\hline Mais de três vezes/semana & 51 & 12,0 \\
\hline \multicolumn{3}{|l|}{ Tipo } \\
\hline Caminhada & 137 & 32,3 \\
\hline Pedalada & 7 & 1,7 \\
\hline Natação & 1 & 0,2 \\
\hline Ginástica & 26 & 6,1 \\
\hline Outra & 21 & 5,0 \\
\hline \multicolumn{3}{|l|}{ Tempo de prática } \\
\hline$\geq 6$ meses & 152 & 35,8 \\
\hline$\leq 6$ meses & 40 & 9,4 \\
\hline \multicolumn{3}{|l|}{ Duração } \\
\hline$\geq 45$ minutos & 132 & 31,1 \\
\hline$\leq 45$ minutos & 60 & 14,1 \\
\hline \multicolumn{3}{|l|}{ Medicamentos } \\
\hline $\operatorname{Sim}$ & 321 & 75,7 \\
\hline Não & 103 & 24,3 \\
\hline \multicolumn{3}{|l|}{$\mathrm{N}^{o}$ medicamentos consumidos/dia } \\
\hline 1 a 3 & 229 & 71,3 \\
\hline Mais de 3 & 92 & 28,7 \\
\hline
\end{tabular}


$\mathrm{Na}$ tabela 3, observa-se que tanto para o sexo feminino quanto masculino, a maioria não tinha o hábito de praticar atividade física; da mesma forma, para ambos os sexos prevaleceu a faixa etária dos 60 aos 70 anos para os que praticavam. Para a escolaridade, observou-se que as mulheres entre 60 e 70 anos tinham tempo de estudo maior ou igual a cinco anos $(25,2 \%)$, no restante das faixas etárias e em ambos os sexos, observou-se escolaridade inferior a cinco anos. Constatou-se também que quanto mais idosos eram os pesquisados, menor era sua escolaridade.

Tabela 3. Associação da faixa etária e sexo com atividade física e escolaridade em idosos. Palmeira das Missões-RS, 2010-2011.

\begin{tabular}{|c|c|c|c|c|c|c|c|c|}
\hline & Sexo & & $\begin{array}{c}60 \mid-65 \\
\mathrm{n}(\%)\end{array}$ & $\begin{array}{c}65 \mid-70 \\
\mathrm{n}(\%)\end{array}$ & $\begin{array}{c}70 \mid-75 \\
\mathrm{n}(\%)\end{array}$ & $\begin{array}{c}75 \mid-80 \\
\mathrm{n}(\%)\end{array}$ & $\begin{array}{c}80 \text { ou mais } \\
\mathrm{n}(\%)\end{array}$ & $\begin{array}{l}\text { Total } \\
\mathrm{n}(\%)\end{array}$ \\
\hline \multirow{6}{*}{ 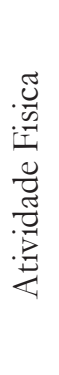 } & \multirow[b]{2}{*}{$\mathrm{F}^{*}$} & $\operatorname{Sim}$ & $35(12,7)$ & $30(10,9)$ & $28(10,2)$ & $20(7,3)$ & $25(9,1)$ & $138(50,2)$ \\
\hline & & Não & $34(12,4)$ & $37(13,5)$ & $29(10,5)$ & $17(6,2)$ & $20(7,3)$ & $137(49,8)$ \\
\hline & \multirow{4}{*}{$\mathrm{M}^{*}$} & Total & $69(25,1)$ & $67(24,4)$ & $57(20,7)$ & $37(13,5)$ & $45(16,4)$ & $275(100)$ \\
\hline & & $\operatorname{Sim}$ & $9(7,4)$ & $19(15,6)$ & $8(6,6)$ & $10(8,2)$ & $8(6,6)$ & $54(44,3)$ \\
\hline & & Não & $18(14,8)$ & $18(14,8)$ & $13(10,7)$ & $10(8,2)$ & $9(7,4)$ & $68(55,7)$ \\
\hline & & Total & $27(22,1)$ & $37(30,3)$ & $21(17,2)$ & $20(16,4)$ & $17(13,9)$ & $122(100)$ \\
\hline \multirow{6}{*}{ 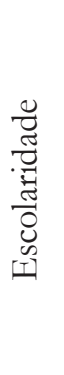 } & \multirow{3}{*}{$\mathrm{F}^{* *}$} & $<5$ anos & $28(9,7)$ & $43(14,8)$ & $38(13,1)$ & $26(9,0)$ & $28(9,7)$ & $163(56,2)$ \\
\hline & & $\geq 5$ anos & $44(15,2)$ & $29(10,0)$ & $21(7,2)$ & $14(4,8)$ & $19(6,6)$ & $127(43,8)$ \\
\hline & & Total & $72(24,8)$ & $72(24,8)$ & $59(20,3)$ & $40(13,8)$ & $47(16,2)$ & $290(100)$ \\
\hline & \multirow[t]{3}{*}{$\mathrm{M}^{*}$} & $<5$ anos & $19(14,2)$ & $25(18,7)$ & $15(11,2)$ & $17(12,7)$ & $16(11,9)$ & $92(68,7)$ \\
\hline & & $\geq 5$ anos & $10(7,5)$ & $15(11,2)$ & $9(6,7)$ & $5(3,7)$ & $3(2,2)$ & $42(31,3)$ \\
\hline & & Total & $29(21,6)$ & $40(29,9)$ & $24(17,9)$ & $22(16,4)$ & $19(14,2)$ & $134(100)$ \\
\hline
\end{tabular}

*Teste qui-quadrado com $\mathrm{p}>0,05 ; * *$ teste qui-quadrado com $\mathrm{p}<0,05$.

\section{DISCUSSÃO}

A perspectiva de envelhecimento populacional modifica a participação dos grupos na vida econômica, política e social do país. É importante conhecer as condições de vida, de saúde, econômicas e de suporte social dos idosos, para que se possa estar preparado para atender às demandas sociais, sanitárias, econômicas e afetivas dessa parcela da população, que é a que mais cresce atualmente. ${ }^{13}$

O envelhecimento populacional brasileiro é um desafio para a saúde pública, pois ao contrário do que acontece nos países desenvolvidos, onde o processo ocorre de forma gradual, acompanhado de melhorias na cobertura do sistema de saúde, condições de habitação, saneamento básico, trabalho e alimentação, no Brasil ocorre rapidamente e num contexto de desigualdades sociais, economia frágil, crescentes níveis de pobreza, com precário acesso aos serviços de saúde e reduzidos recursos financeiros, sem as modificações estruturais que respondam às demandas do novo grupo etário emergente. ${ }^{14}$

$\mathrm{Na}$ análise dos dados sociodemográficos dos idosos pesquisados, observou-se que a maioria é do sexo feminino, o que está de acordo com o estudo de Fernandes, ${ }^{15}$ que encontrou 61,5\%. 
Também segundo o IBGE, ${ }^{3}$ a população idosa feminina corresponde a 55\%, referendando a feminilização da velhice. As mulheres predominam entre a população idosa, dada a menor mortalidade, realidade de muitos países, com uma taxa maior em países desenvolvidos. ${ }^{14}$ A sobremortalidade masculina, tanto de adultos e idosos jovens, e a consequente "feminização do envelhecimento" resultam das maiores taxas de mortalidade por doenças circulatórias, doenças relacionadas ao tabagismo, alcoolismo e causa externas como mortes violentas e atropelamentos, entre os homens. ${ }^{14}$

Ainda no Brasil, deve-se considerar o importante aumento da população idosa de 70 anos ou mais de idade. Em 1999 correspondia a 3,9\%, e em 2009 atingiu 5,1\%. No Rio Grande do Sul, os idosos acima de 70 anos correspondem a 6,1\% da população gaúcha, e entre a população idosa corresponde a 44,6\%, de acordo com o Censo do IBGE de $2010 .{ }^{16} \mathrm{O}$ crescimento acelerado, no Brasil, do número de idosos mais velhos, demanda um redirecionamento das políticas públicas em prol da implementação de estratégias adequadas às necessidades da população. A atenção à saúde da população idosa deve pautada nos princípios do Sistema Único de Saúde, visando à integralidade da assistência e na perspectiva da manutenção da autonomia e capacidade funcional. ${ }^{17}$

Com a redução da mortalidade em idade mais avançada, aumentou o número de idosos com maior fragilidade. A Política Nacional de Atenção à Saúde do Idoso, baseando-se num conceito mais genérico de fragilidade, considera dentro da classificação de idosos frágeis também aqueles maiores de 75 anos. ${ }^{18}$ Embora a fragilidade, no sentido mais estrito do termo, seja considerada síndrome ainda sem definição consensual, sabese que ela pode interferir na funcionalidade dos idosos, causando, em geral, maior dependência. ${ }^{19}$ A síndrome da fragilidade tem maior prevalência na idade mais avançada. ${ }^{20}$ Contudo, os idosos demandam cuidados mais específicos, sendo necessário capacitar os profissionais de saúde no seu cuidado e adequar os serviços de saúde para atender satisfatoriamente a esse contingente populacional. $^{21}$
O estado civil predominante foi "casado", seguido de "viúvo"; valores próximos foram encontrados no estudo de Fernandes, ${ }^{15}$ que foi de $52 \%$ para casados e $38 \%$ para viúvos. Em estudo de Pilger et al., ${ }^{22}$ dentre os idosos estudados, a maioria (54\%) tem menos de cinco anos de estudo. No Brasil, a escolaridade dos idosos brasileiros é ainda considerada baixa, ou seja, $30,7 \%$ tinham menos de um ano de instrução no censo 2010 do IBGE. ${ }^{16}$ E segundo estudo de Aires et al., ${ }^{17}$ na população idosa atual observase, ainda, alto índice de analfabetismo, associado, entre outros fatores, à dificuldade de acesso à escola, principalmente fora dos grandes centros urbanos, visto que entre os idosos da região rural houve maior proporção de analfabetismo ou com menos de quatro anos de estudo.

A falta de instrução entre os idosos possui estreita relação com dificuldades no trato da saúde por parte dos mesmos, a exemplo de problemas com manuseio de medicamentos, seguimento de dietas, prescrições e outros. Isso requer dos profissionais de saúde maior atenção relativa ao tipo e forma de linguagem que devem nortear o processo de comunicação entre eles e os idosos na operacionalização do cuidado terapêutico. ${ }^{15}$

Constatou-se renda mensal dos idosos relativamente baixa, mas condizente com a da maioria dos idosos do Brasil, tendo as aposentadorias e pensões como fontes predominantes. Valores semelhantes foram encontrados nos estudos de Fernandes, ${ }^{15}$ onde $88 \%$ dos idosos que possuem renda de um salário mínimo proveniente de aposentadoria ou pensão.

A maioria dos idosos pesquisados não faz parte da População Economicamente Ativa (PEA); sendo assim, o benefício recebido pelo idoso proveniente da Previdência Social, na forma de aposentadoria e pensão, cumpre a função de proteção social importante. Por meio dele, é possível constatar, no espaço familiar, uma revalorização da pessoa idosa que, de posse da renda oriunda de sua aposentadoria, obtém uma espécie de salvaguarda de subsistência familiar. Dessa forma, os idosos invertem o papel social de assistido para assistente. ${ }^{23}$ No entanto, dois 
em cada 100 idosos podem ser considerados indigentes, e 11 em cada 100 encontram-se abaixo da linha de pobreza. Destaque deve ser dado para a importância de políticas públicas e sociais na garantia efetiva dos direitos dos idosos visando a um envelhecimento digno, de forma que possa suprir suas necessidades físicas, biológicas, psicológicas, socais e nutricionais. É sabido, no entanto, que muitos idosos ainda estão à margem do alcance destas políticas de Estado, o que faz perdurar a situação de injustiça social e, consequentemente, a insegurança alimentar do idoso. ${ }^{24}$

A pesquisa revelou que os maiores gastos dos idosos são com alimentação. É interessante mencionar, segundo estudos de Almeida, ${ }^{25}$ que em relação ao consumidor idoso, constata-se a existência de uma preocupação crescente com a saúde, o que o conduz à busca por alimentos mais saudáveis, tornando o estudo da alimentação do idoso bastante relevante. $\mathrm{O}$ crescimento da população idosa representa um grande mercado de consumo alimentar, fato que exige adequação dos alimentos, para permitir maior tempo de vida saudável para esses consumidores. Consequentemente, isso gera uma redução de custos para o estado e a sociedade, pois um melhoramento na condição de vida, por meio da alimentação mais saudável, pode auxiliar na prevenção de doenças e manutenção da saúde de seus idosos.

No presente estudo, os gastos com medicamentos e saúde também foram expressivos, e a maioria relatou fazer uso de um a três medicamentos por dia. Isto preocupa, pois o uso de medicamentos por idosos traz como consequência um equilíbrio muito delicado entre risco e benefício; assim, os mesmos medicamentos que podem prolongar a vida do idoso podem custar a sua qualidade de vida.

A vulnerabilidade dos idosos às reações adversas ao consumo de medicamentos é muito alta, devido àcomplexidade dos problemas clínicos, à necessidade de múltiplos agentes e às alterações farmacocinéticas e farmacodinâmicas inerentes ao envelhecimento. Nesse contexto, um dos grandes desafios nos serviços de saúde é contribuir na promoção do uso racional dos medicamentos. Algumas estratégias que podem ajudar a prevenir e minimizar os efeitos adversos dos medicamentos incluem a educação dos usuários, especialmente quanto à prática da automedicação, incluindo o uso de fitoterápicos; o aconselhamento sobre os riscos da interrupção, troca, substituição ou inclusão de medicamentos sem conhecimento dos profissionais da saúde e o aprazamento rigoroso dos horários indicados na prescrição médica, evitando a administração simultânea de medicamentos que podem interagir entre si ou com alimentos. ${ }^{26}$

No presente estudo, a grande maioria dos idosos não consumia bebidas alcoólicas. Fator positivo, visto que o alcoolismo configura importante problema de saúde pública, uma vez que os idosos muitas vezes se tornam mais vulneráveis ao uso de álcool e tabaco. Pelo fato de a população idosa fazer parte de um grupo que apresenta vários problemas de saúde, o consumo de muitos medicamentos que, combinados com as substâncias nocivas presentes no fumo e álcool, tornam os idosos mais susceptíveis a interações tanto medicamentosas quanto com os alimentos, agrava o quadro instalado, causando dificuldade de recuperação e interação social. ${ }^{27}$

Outro dado importante para avaliar a saúde dos idosos é o tabagismo, sendo que a maioria dos pesquisados não possuía esse hábito. O tabagismo é apontado como uma das principais causas de doenças e morte em boa parte do mundo, desfavorecendo a longevidade, sendo fator de risco para inúmeras doenças, principalmente câncer, doenças cardiovasculares e respiratórias. ${ }^{28}$

Um grande contingente de idosos neste estudo não possuía o hábito de realizar atividade física, fator preocupante, visto que está comprovado que a inatividade acarreta problemas não somente físicos, como o surgimento de doenças crônicas e de ordem mental. O indivíduo, ao envelhecer, passa por um processo natural de mudanças que é particular de cada um, mas inevitável, sendo influenciado positiva ou negativamente 
pelas mudanças do estilo de vida, em especial pela diminuição da capacidade física e também decorrente da aposentadoria. ${ }^{29}$

A prática regular de atividade física aumenta a capacidade de realizar as atividades da vida diária, diminuindo a dependência, prolongando sua independência, aumentando a autoestima e, consequentemente, contribuindo para uma melhor qualidade de vida. Enfim, os aspectos positivos e negativos da saúde mental podem se beneficiar do exercício físico regular, tornando o idoso menos dependente de medicamentos para se sentir bem. ${ }^{29}$ Embora as atividades físicas não devam ser entendidas como receitas prontas, pois há necessidade de levar em consideração a individualidade biológica, idade, sexo, estado de saúde, objetivos e preferências dos indivíduos, há a recomendação da prática de atividade física tanto na prevenção como no tratamento de doenças. ${ }^{30}$

A perspectiva do envelhecimento populacional gera muitas preocupações. Segundo estudos de Portugal, ${ }^{31}$ o aumento do número de idosos e o declínio da proporção de jovens resultarão em desequilíbrio, causado pela quantidade de pessoas ativas e inativas. $\mathrm{O}$ impacto que isso gera no sistema de segurança social e nos sistemas de saúde são os serviços mais procurados, e deverão cada vez mais cumprir um papel de longa duração, gerando um desafio muito grande também na formação técnica e profissional na área da saúde. Os idosos são mais dependentes desses serviços, em especial quando se refere às idosas, visto que as mulheres vivem mais tempo, ficam solitárias, viúvas - enfim, mais vulneráveis. Toda essa realidade precisará refletir em políticas públicas que possam atender a essas novas demandas, pois o maior desafio está em construir uma sociedade para todas as idades, promovendo a participação dos idosos, desenvolvendo políticas inclusivas, combatendo as desigualdades, aproveitando o potencial das famílias e dando-lhes suporte.

No presente estudo, as limitações metodológicas referem-se ao fato de não ser possível descrever claramente o que é causa e o que é efeito, por se tratar de um estudo com delineamento do tipo transversal, necessitando para isso de pesquisas do tipo longitudinal.

\section{CONCLUSÃO}

O perfil sociodemográfico ficou constituído, principalmente, de idosos do sexo feminino, de 65 a 70 anos, casados, menos de cinco anos de escolaridade, com renda de um a dois salários mínimos, provenientes da aposentadoria e pensão. Desta renda dependem duas pessoas e as principais despesas são para alimentação e saúde/medicamentos.

Quanto aos hábitos de vida, a maioria dos idosos não consome bebida alcoólica e não é tabagista, mas os que fumam já o fazem por mais de 35 anos. A atividade física não é uma prática regular e quem o faz pratica apenas uma vez por semana, mais de 45 minutos, sendo a caminhada a opção mais citada e o tempo de prática mais de seis meses. Quanto ao consumo de medicamentos, há predominância de um a três medicamentos ao dia.

No entanto, conhecendo melhor o perfil desta população idosa, é importante ressaltar a necessidade de políticas públicas para atender às novas demandas, sendo um grande desafio para a gestão pública. Dentre esses desafios encontramse, ainda: capacitar técnicos e profissionais em saúde no planejamento do cuidado integral ao idoso, dentro de uma rede de atenção ao envelhecimento com forte regulação pela atenção primária à saúde; desenvolver políticas inclusivas, valorizando mais a pessoa do idoso, assim como seus cuidadores/família, oferecendo suporte adequado, tanto formal quanto informal.

A meta na gestão pública deve ser a manutenção da capacidade funcional do idoso; assim, uma das estratégias é a criação de programas de atividades físicas, com a disponibilidade não somente de equipamentos, mas especialmente da orientação de um profissional da área de Educação Física. Este tipo de programa visaria combater o sedentarismo, comum nesta faixa etária, além de estimular a inserção social e o desempenho físico funcional. Enfim, é importante ter um novo olhar sobre o perfil populacional que a cada dia está envelhecendo mais e acompanhar esse processo, de modo que sejam atendidas as necessidades da forma mais satisfatória possível. 


\section{REFERÊNCIAS}

1. Veras RP. Envelhecimento populacional contemporâneo: demandas, desafios e inovações. Rev Saúde Pública 2009;43(3):548-54.

2. Closs VE, Schwanke CHA. A evolução do índice de envelhecimento no Brasil, nas suas regiões e unidades federativas no período de 1970 a 2010. Rev Bras Geriatr Gerontol 2012;15(3):443-58.

3. Instituto Brasileiro de Geografia e Estatística. Primeiros resultados definitivos do Censo de 2010 [Internet]. Rio de Janeiro: IBGE; c2014 [acesso em 25 Set. 2012]. Disponível em: http://www.ibge.gov.br/ home/presidencia/noticias/noticia_visualiza.php?id_ noticia $=1866 \&$ id_pagina $=1$.

4. Hunter RH, Anderson LA, Belza B, Bodiford K, Hooker SP, Kochtitzky CS, et al. Environments for healthy aging: linking prevention research and public health practice. Prev Chronic Dis 2013;10:E55.

5. The maturing of America: communities moving forward for an aging population [Internet]. Washington: National Association of Area Agencies on Aging; 2011 [acesso 21 mar 2014]. Disponível em http://www.n4a.org/files/MOA_FINAL_Rpt.pdf.

6. Veras RP, Caldas CP, Coelho FD, Sanchez MA. Promovendo a Saúde e Prevenindo a Dependência: identificando indicadores de fragilidade em idosos independentes. Rev Bras Geriatr Gerontol 2007;10(3):355-70

7. Motta LB, Aguiar AC, Caldas CP. Estratégia saúde da família e a atenção ao idoso: experiências em três municípios brasileiros. Cad Saúde Pública 2011;27(4):779-86.

8. Martins JJ, Schier J, Erdmann AL, Albuquerque GL. Políticas públicas de atenção à saúde do idoso: reflexão acerca da capacitação dos profissionais da saúde para o cuidado com o idoso. Rev Bras Geriatr Gerontol 2007;10(3):371-87.

9. Barbetta PA. Estatística aplicada às ciências sociais. 7. ed. Florianópolis: UFSC; 2007.

10. Duncan BB, Chambless LE, Schidt MI, Folsom AR, Szklo M, Crouse Jr 3, et al. Association of the waist-to-hip ratio is different with wine than with beer or hard liquor consumption. Am J Epidemiol 1995;142(10):1034-8.

11. Witteman, JCM, Grobbee DE, Valkenburg HA, van Hemert AM, Stijnen T, Hofman A. Cigarrette smoking and the development and progression of aortic atherosclerosis. Circulation 1993;88(5 Pt 1):2156-62.

12. Haskell WL, Lee I, Pate LL, Powell KE, Blair SN, Franklin BA, et al. Physical Activity and Public Health: updated Recommendation for Adults From the American College of Sports Medicine and the American Heart Association. Circulation 2007;116(9):1081-93.

13. Brasil. Secretaria de direitos Humanos. Informe Brasil para a III Conferência Regional Intergovernamental sobre Envelhecimento na América Latina e Caribe [Internet]. Costa Rica, 2012 [acesso 12 mar 2014]. Disponível em: http://www.cepal.org/celade/ noticias/paginas/9/46849/Brasil.pdf.

14. Chaimowicz F. Saúde do idoso. 2. ed. Belo Horizonte: NESCON UFMG; 2013.

15. Fernandes MGM, Souto MC, Costa SFG, Fernandes BM. Qualificadores sócio-demográficos, condições de saúde e utilização de serviços por idosos atendidos na atenção primária. Rev Bras Ciênc Saúde 2009;13(2):13-20.

16. Instituto Brasileiro de Geografia e Estatística. Síntese de Indicadores Sociais: uma análise das condições de vida da população brasileira. Rio de Janeiro: IBGE; 2010.

17. Aires M, Paskulin LMG, Morais EP. Capacidade funcional de idosos mais velhos: estudo comparativo em três regiões do Rio Grande do Sul. Rev Latinoam Enferm 2010;18(1):11-7.

18. Brasil. Ministério da Saúde. Portaria n. 2528, de 19 de outubro de 2006. Aprova a Política Nacional de Saúde da Pessoa Idosa. Brasília ,DF: Ministério da Saúde; 2006 [acesso 18 Out. 2012]. Disponível em http:// www.ciape.org.br/PoliticaPIdosa2528.pdf

19. Pavarini SCI, Barha EJ, Mendiondo MSZ, Filizola CLA, Filho JFP, Santos AA. Família e vulnerabilidade social: um estudo com octogenários. Rev Latinoam Enferm 2009; 7(3):91-6.

20. Veras R. Experiências e tendências internacionais de modelos de cuidado para com o idoso. Ciênc Saúde Coletiva 2012;17(1):231-8.-

21. Sampaio LS, Neto DGS, Reis LA, Lauton MAR, Reis LA, Santos AO, et al. Condições sociodemográficas e de saúde de idosos residentes em domicílio no município de Jequié-BA. Rev Bras Geratr Gerontol 2009;12(2):267-74.

22. Pilger C, Menon MH, Mathias TAF. Características sociodemográficas e de saúde de idosos: contribuições para os serviços de saúde. Rev Latinoam Enferm 2011;19(5):1230-38.

23. Areosa SVC, Areosa AL. Envelhecimento e dependência: desafios a serem enfrentados. Textos Contextos 2008;7(1):138-50.

24. Silva PDN, Kernkamp CL, Bennemann RM. Insegurança alimentar: as desigualdades de renda e a vulnerabilidade social como resultados na 
alimentação dos idosos. Rev. UNINGÁ Review 2013;16(2):38-43.

25. Almeida IC, Guimarães GF, Rezende DC, Sette RS. Hábitos alimentares da população idosa: padrões de compra e consumo. In: Programa de Pós-Graduação em Administração da FEA-USP. Seminário em administração. $8^{\circ}$ SEMEAD: sustentabilidade Ambiental nas Organizações; 09-10 Set. 2010; São Paulo: USP; 2010.

26. SecoliI SR. Polifarmácia: interações e reações adversas no uso de medicamentos por idosos. Rev Bras Enferm 2010;63(1):136-40.

27. Senger AEV, Ely LS, Gandolfi T, Schneider RH, Gomes I, De Carli GA. Alcoolismo e tabagismo em idosos: relação com ingestão alimentar e aspectos socioeconômicos. Rev Bras Geriatr Gerontol 2011;14(4):713-9.
28. Goulart D, Engraff P, Ely LS, Sgnaolin V, Santos EF, Terra NL, et al. Tabagismo em idosos. Rev Bras Geriatr Gerontol 2010;13(2):313-20.

29. Mittelmann C. Efeitos da atividade física na saúde mental do idoso: estudo de revisão de artigos científicos [dissertação]. Porto alegre: Universidade Federal do Rio Grande do sul; 2010.

30. Coelho CF, Burini RC. Atividade física para prevenção e tratamento das doenças crônicas não transmissíveis e da incapacidade funcional. Rev Nutr 2009;22(6):937-46.

31. Portugal S. Envelhecimento: um desafio ao futuro [Internet]. Centro de Estudos Sociais. Coimbra: CES; 2008 [Acesso em 10 Out. 2012]. Disponível em: http://www.ces.uc.pt/destaques/ecp2008/SP.pdf 